\title{
Experimental Comparison of Antenna Clustering Strategies in MIMO Distributed Antenna Systems
}

\author{
George S.D. Gordon, Michael J. Crisp, Richard V. Penty and Ian H. White \\ Centre for Photonic Systems, Electrical Division, Department of Engineering, University of Cambridge \\ 9 JJ Thomson Avenue, Cambridge, CB3 0FA, United Kingdom \\ gsdg2@cam.ac.uk
}

\begin{abstract}
In this paper the effect of partitioning arrays of transmitting antennas into spatially separated clusters on the condition number and capacity of MIMO wireless systems is examined using experimental channel measurements of an indoor MIMO-enabled distributed antenna system $(D A S)$. It is first shown for a $3 \times 2$ MIMO system that distributing the transmit antennas into spatially separated clusters provides an improvement in channel conditioning $(\sim 1 \mathrm{~dB})$ and hence capacity, in line with previous findings. Next, a configuration with 6 transmit antennas and 2 receive antennas is examined and it is found that when it is operated as a $6 \times 2$ MIMO system, it makes negligible difference to the conditioning of the channel whether the transmit antennas are grouped into 3 clusters of 2 antennas or 2 clusters of 3 antennas. The capacity varies by only a small amount $(\sim \% 1)$ between the two clustering schemes, which can be accounted for by environment-specific signal-tonoise ratio (SNR) effects. It is then concluded that for the two typical indoor DAS scenarios tested here, if sufficient transmit diversity is provided (i.e. there are a relatively large number of transmit antennas compared to receive antennas), greater spatial distribution through increased clustering provides diminishing performance improvements. Given the typically lower installation cost of less distributed arrangements, they may then be a preferred option in commercial DAS deployments.
\end{abstract}

\section{INTRODUCTION}

The proliferation of wireless devices coupled with society's increased reliance on mobile services has lead to vast increases in mobile data usage, with a predicted 13-fold growth from 2012 to 2017 [1]. This places increasing strain on wireless networks, particularly inside buildings where up to $90 \%$ of mobile data traffic is estimated to originate [2]. Many new wireless protocols have been developed to increase the capacity of networks so that they can meet this demand. One of the most prominent protocol improvements has been multipleinput multiple-output (MIMO) in which arrays of transmit and receive antennas are used to perform spatial multiplexing over the wireless channel. This has enabled substantial increases in available capacity and has been incorporated into most current and emerging wireless protocols, including 4G/LTE, 5G, IEEE 802.11n and 802.11ac.

Improvements in wireless protocols can also be enhanced further by improved infrastructure designs. One notable example is distributed antenna systems (DAS), in which the antennas of a wireless base station are replicated and distributed around a coverage area, as opposed to conventional collocated antenna systems $(C A S)$. Typically, the base station is located at a central hub, and the RF antenna feeds are transported directly to remote antenna units (RAUs), where they are amplified and re-transmitted as illustrated in Figure 1. The reverse process occurs on the uplink. The cables used to feed the RAUs are usually either coaxial or optical fibre, with the latter making use of radio-over-fiber (RoF) technology. RoF systems typically amplitude modulate the RF signal onto a laser at the transmitter and then demodulate it using a photodiode at the receiver to recover the original signal. Such systems can exploit the flat spectral profile of fibres over large optical bandwidths, enabling the distribution of broadband, multiservice RF feeds. DAS have been shown to improve in-building coverage for a range of wireless services and as a result are a popular technology, with over 89,000 installations worldwide [3].

More recently, it has been shown that DASs can be used in conjunction with MIMO-enabled wireless systems to provide greater improvements in capacity than either technology alone [4]. Distributing RF MIMO streams, either by replicating the streams at every RAU or by sending each stream to a separate RAU, has been shown to offer improved capacity compared to MIMO CAS systems [5]. If the streams are replicated at multiple RAUs this results in an excess of transmit antennas, which provides another avenue to increase capacity by application of beamforming algorithms (provided channel state information can be fed back to the transmitter) [6]. Large numbers of transmit antennas are an increasingly common feature of emerging wireless protocols, such as 802.11ac which can have up to 8 transmit antennas. Even greater numbers of transmit antennas are seen in large scale antenna systems (LSAS), which have recently gained popularity due to their ability to form highly focussed RF beams, enabling higher channel capacities with lower energy usage [7].

In all the above cases, there are many different ways in which the large number of transmit antennas could be partitioned into distributed groups of collocated antennas, termed clusters here. An example of this is shown in Figure 1. From a coverage perspective, it is well known that for the same coverage area it is best to distribute antennas as evenly as possible in order to optimise the mean received signal power [8]. Theoretical work has found that for a fixed number of transmit antennas there is no improvement in ergodic channel capacity gained by further distributing antennas when the size of each cluster becomes less than or equal to the number of receive antennas [9]. It has also been shown theoretically that for a fixed number of transmit antennas, better capacity can be achieved when using a limited clustering scheme, i.e. 2 clusters of 2 transmit antennas can provide higher capacity than 4 clusters of single antennas or 1 cluster of 4 [10]. However, there has been little experimental work to examine the effect 


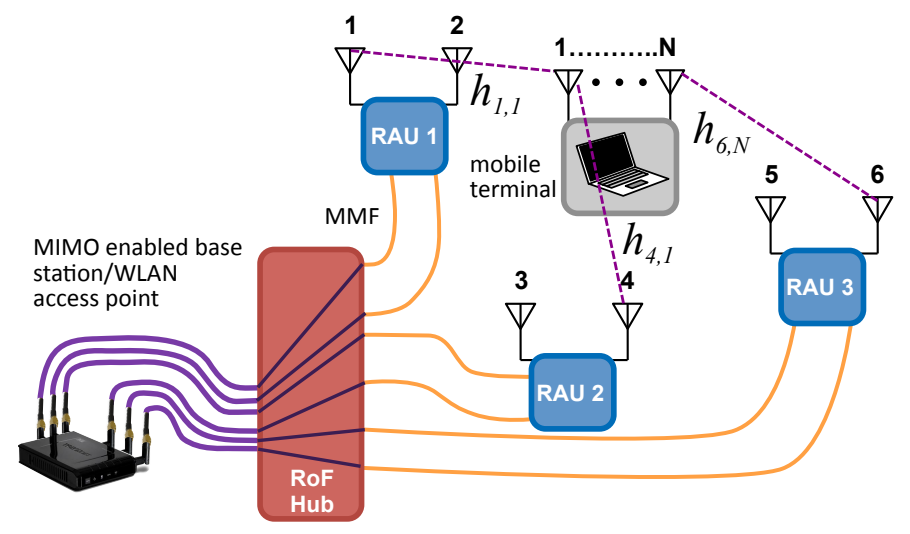

(a)



(b)

Fig. 1.

of clustering for a fixed number of transmit antennas in real indoor propagation scenarios.

In this paper, experimental channel measurements for two typical indoor DAS scenarios are used to a compare the channel conditioning and capacity of two transmit antenna clustering strategies: 3 clusters of 2 antennas and 2 clusters of 3 antennas. The two propagation environments represent two quite different situations found inside buildings - one has significant line-of-sight (LOS) propagation, and the other has only non-line of sight (NLOS) propagation.

It is first found, in agreement with previous results, that for a $3 \times 2$ MIMO system, distributing the 3 transmit streams individually improves the median condition number by $\sim 1 \mathrm{~dB}$ and capacity by $\sim 3 \%$ for a typical indoor wireless set-up. Next, a system with 6 transmit antennas is tested in the two clustering configurations mentioned previously. When examined as a $6 \times 2$ MIMO system, it is found that the 3 -cluster case has a condition number almost identical to that of the 2-cluster case. The capacity is also noted not to differ significantly between the two cases $(<1 \%)$, with the difference likely being due to environment-specific SNR effects.

This suggests that in an indoor MIMO DAS with a fixed number of transmit antennas, the exact clustering scheme used may have a diminished effect on channel conditioning and capacity provided sufficient transmit diversity is available. Although this has been examined theoretically, as far as the authors are aware this is the first experimental data presented examining this effect in an indoor setting.

\section{THEORY}

For a MIMO channel with $M$ transmit and $N$ receive antennas, the propagation channel is represented by a complex $N \times M$ matrix, $\mathbf{H}$. In this work it is assumed that because the propagation environments tested are very small, the multipath delay spread is relatively small (of the order of 10-30ns) and so the coherence bandwidth is on the order of $10-50 \mathrm{MHz}$. This is comparable to the channel bandwidth of $802.11 \mathrm{~g} / \mathrm{n}$ of $20 \mathrm{MHz}$ and so $\mathbf{H}$ is assumed to have a relatively flat frequency response, i.e., to be a flat-fading channel [11]. For a flat-fading channel with no channel state information (CSI) fed back to the transmitter, as is the case in most current wireless protocols, the Shannon channel capacity is known to be [12]:

$$
C=B \sum_{p=1}^{S} \log _{2}\left(1+\frac{\rho}{M} \sigma_{p}^{2}\right)
$$

where $C$ is the channel capacity in bit/s/Hz, $S$ is the rank of $\mathbf{H}, \rho$ is the total SNR of the system and $\sigma_{p}$ is the $p^{\text {th }}$ largest magnitude singular value of $\mathbf{H}$. This can be thought of as the sum of the capacities of several eigenchannels of $\mathbf{H}$, each of which has an SNR scaled by a singular value of $\mathbf{H}$. The distribution of these singular values is clearly important to the capacity of this channel - the more even the distribution, the higher the potential capacity. This measure is well encapsulated by the condition number:

$$
K=20 \log _{10}\left(\frac{\max _{p}\left(\sigma_{p}\right)}{\min _{p}\left(\sigma_{p}\right)}\right)
$$

where $K$ is the condition number in $\mathrm{dB}, \sigma_{p}$ represents the $p^{\text {th }}$ singular value of the channel matrix $\mathbf{H}, \max _{p}\left(\sigma_{p}\right)$ represents the maximum $\sigma_{p}$ across all $p$, and $\min _{p}\left(\sigma_{p}\right)$ represents the minimum $\sigma_{p}$ across all $p$. Thus, the condition number represents the degree to which the wireless propagation channel is able to be multiplexed - the lower it is, the higher the achievable spatial multiplexing gain. As a result, in this work the condition number is considered to be a fundamental indicator of how a different antenna clustering scheme impacts the performance of MIMO.

It should be noted that the channel matrix, $\mathbf{H}$, varies randomly due to multipath fading - in fact this a key requirement need in order for MIMO to work. As a result, there is a random distribution of channel capacities and of condition numbers. In this work, a range of different fading scenarios are tested and hence the results are presented in terms of probabilistic distributions and their median values.

\section{EXPERIMENTAL SET-UP}

In order to evaluate channel capacity and conditioning, the channel matrix, $\mathbf{H}$, is measured experimentally using the setup shown in Figure 2. Only one transmit cluster is tested at a time so it is ensured that the environment is kept absolutely identical when the cluster is moved between measurements.

A vector network analyser (VNA) is used to measure the amplitude and phase of the transfer coefficients between each 




Fig. 2. Experimental set-up of the MIMO channel measurement system. Note that the central antenna is only used when measuring clusters with three antennas.

input port on the DAS and the output port of each receive antenna. Measurements are taken in 1600 frequency channels over a range of $1.7 \mathrm{GHz}$ to $2.7 \mathrm{GHz}$, and are then carefully calibrated to compensate for the frequency dependent gain of the antennas and the RoF links. Through this method, many different multipath fading scenarios are tested, effectively creating a fast-fading environment where each measurement becomes a random sample [13]. In this way, the statistical properties of the channel are able to be estimated as discussed in Section II.

The measurements are taken in two different DAS scenarios - one which has a significant line-of-sight (LOS) propagation component, and the other which has almost entirely nonline-of-sight (NLOS) propagation, shown in figure 3. Both environments are rich in multipath fading, a requirement for MIMO. For each environment, there are 3 possible ways that the 2-cluster case could be implemented. Because of this, each channel matrix sample uses a randomly selected combination of two cluster positions, with the aim of reducing unwanted SNR bias. Similarly, when comparing CAS with DAS, the position of the collocated antennas is selected randomly from the three possible locations.

The DAS used in both cases is a Zinwave 2700 RoF system, operating at $1310 \mathrm{~nm}$ and providing 30m links over OM1 MMF. Offset launch techniques are used to enhance the bandwidthdistance product of the MMF. This is then a broadband system and is specified to provide services from $300 \mathrm{MHz}$ to $3 \mathrm{GHz}$.

In order to determine the channel condition number and capacity for the different clustering schemes, the measured channel matrices are processed as described in section II. Except in the case where the SNR is explicitly varied, the transit power used is $15 \mathrm{dBm}$ and the noise floor is $-90 \mathrm{dBm}$ in a $1 \mathrm{~Hz}$ bandwidth, typical values for $802.11 \mathrm{n}$ compliant access points [14]. The total transmit power is kept constant across all schemes tested.

\section{RESULTS}

Figure 4 compares the cumulative distribution functions (CDF) of the measured channel capacity for a $3 \times 2 \mathrm{MIMO}$ DAS and a CAS. It can be seen that there is about a $2.5 \%$ increase in capacity when going from a CAS to a DAS, which is consistent with previous results presented elsewhere. Figure 5 shows the improvement in condition number, about $1.5 \mathrm{~dB}$, which explains at least of some of the observed capacity improvement.

Figure 6 presents the median measured channel capacity in the LOS scenario for the two different clustering strategies as a function of transmit power. Two types of MIMO systems are considered - a $6 \times 2$ MIMO system using all transmit antennas, and a system in which each cluster is considered as a separate

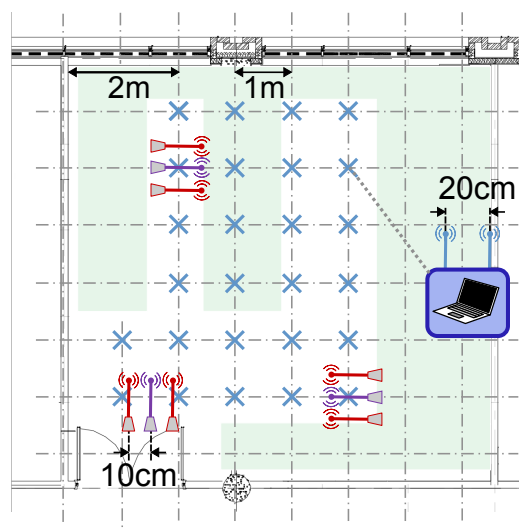

(a)

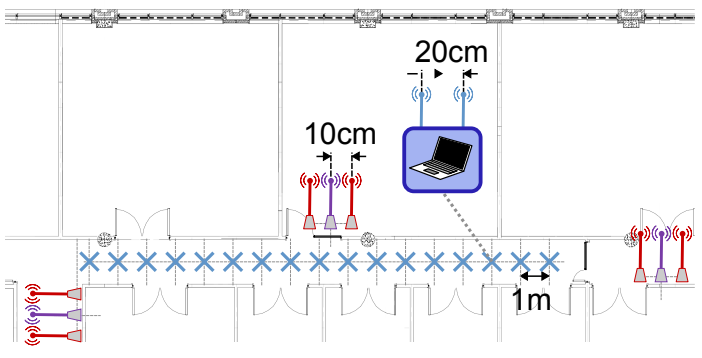

(b)

Fig. 3. The two test propagation environments (a) line-of-sight (LOS) scenario and (b) non-line-of-sight (NLOS) scenario, indicating location of transmit antennas and receiver test points. The central transmit antennas in each case are excluded when testing clusters with only two antennas.

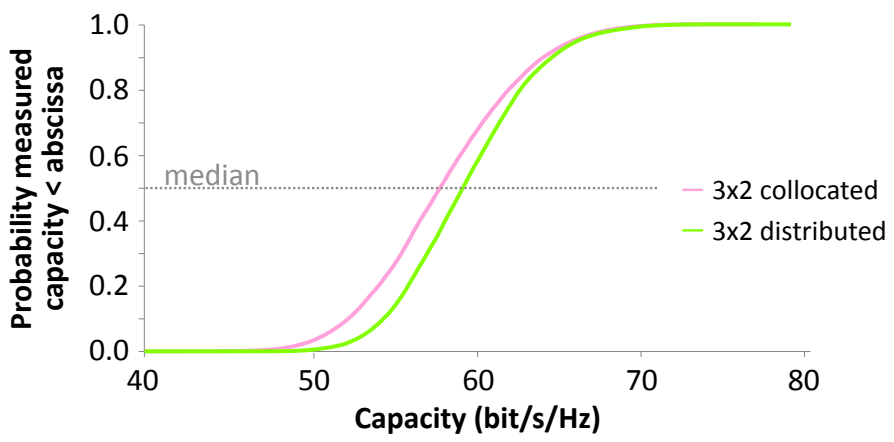

Fig. 4. CDF showing the improvement in capacity offered by switching from a CAS to DAS for a $3 \times 2$ MIMO system with 3 transmit antennas in the LOS propagation scenario.

base station. It is seen that for the $6 \times 2 \mathrm{MIMO}$ case there is only a difference of $<1 \mathrm{~dB} / \mathrm{Hz}$. There is a slightly larger improvement for the separate base-station scenario of around $1.5 \mathrm{~dB} / \mathrm{Hz}$. In both cases, the change is relatively small.

The CDFs of capacity for both the LOS and the NLOS propagation scenarios are shown in Figure 7 . It is seen that the more highly distributed clustering approach (3 clusters of 2) results in a shift of median capacity by $\sim 1 \%$ in both the LOS and NLOS scenarios. This difference is small enough that it is considered negligible, particularly considering the environment specific SNR bias present in both cases. For example, in the the case with 2 clusters of 3 antennas, there is chance that a user in the room would be further away from every antenna cluster than is possible with 3 clusters. However, this is compensated for by the fact that if a user is close to a cluster of antennas 


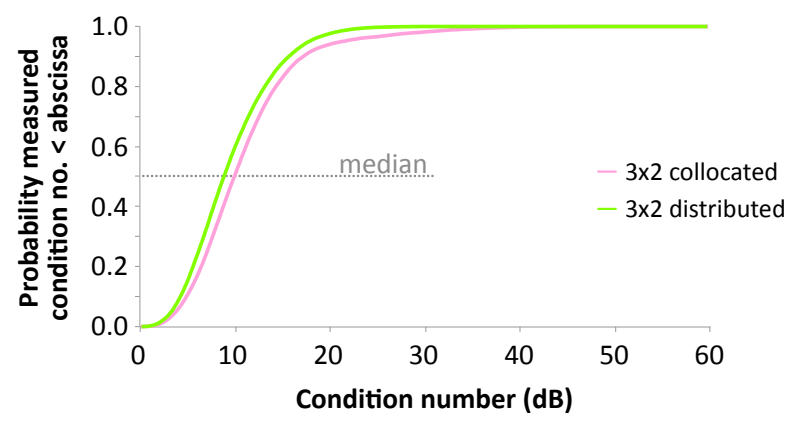

Fig. 5. CDF showing the improvement in condition number offered by switching from a CAS to DAS for a $3 \times 2$ MIMO system with 3 transmit antennas in the LOS propagation scenario.
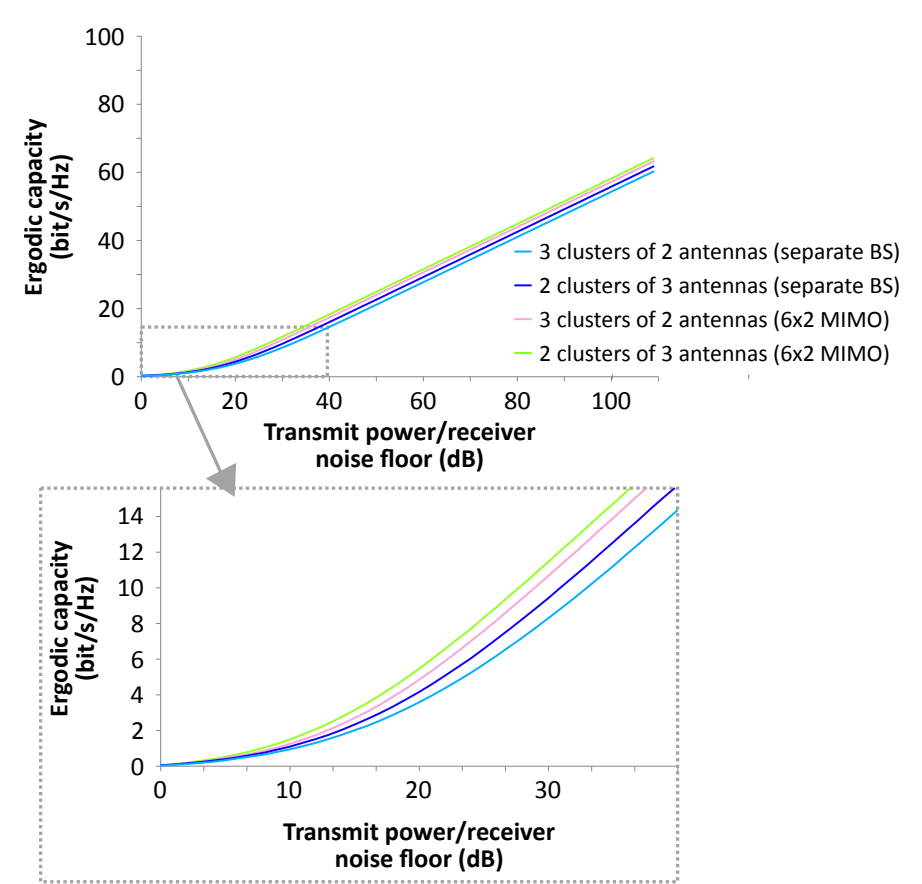

Fig. 6. Median capacity of different clustering strategies as transmit power is increased.

in the 2-cluster case they will receive $3 \mathrm{~dB}$ more power. SNR effects such as this can introduce slight variations to the results and so the curves are considered to differ negligibly.

Further insight is gained from considering the condition number distributions for the LOS and NLOS scenarios, shown in Figure 8. It is seen that for a clustered layout using separate base stations there is a reduction in condition number when using 2 clusters as opposed to 3 . This indicates that the additional diversity obtained from using an extra transmit antenna is able to provide a better conditioned channel. This in turn explains why the capacity improvement is slightly greater for the separate base station case.

However, in the $6 \times 2$ MIMO case, there is no discernible difference in condition number between the two clustering strategies in either the LOS or the NLOS scenarios. This suggests that if the available transmit diversity is fully utilized because of a sufficiently large transmit array, then further distribution of antennas does not improve channel conditioning or capacity in this case. In the case of separate base stations, a more significant improvement is obtained because additional



(a)

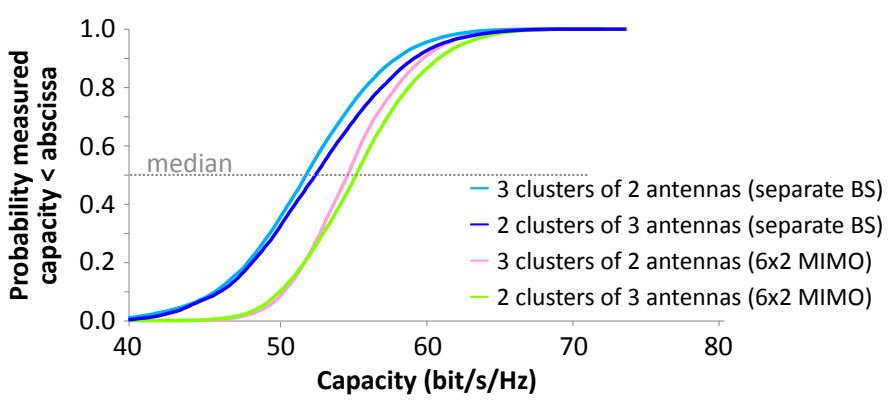

(b)

Fig. 7. CDFs comparing the capacity of different clustering strategies: (a) LOS scenario and (b) NLOS scenario. A transmit power of $15 \mathrm{dBm}$ and a receiver noise floor of $-90 \mathrm{dBm}$ in a $20 \mathrm{MHz}$ bandwidth are assumed.



(a)

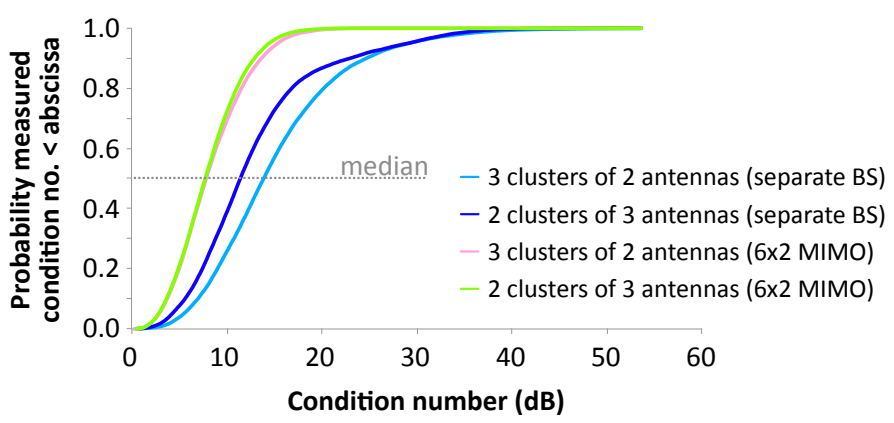

(b)

Fig. 8. CDF of condition number for two scenarios: (a) LOS case and (b) NLOS case

transmit diversity within each base station is introduced.

\section{CONCLUSION}

While distributing transmit antennas in a MIMO DAS can improve capacity by increasing channel diversity, in the two representative indoor propagation scenarios tested here with 6 transmit antennas (tested as a $6 \times 2$ MIMO system), the channel diversity is not significantly different whether the antennas are 
placed in 2 clusters of 3 or 3 clusters of 2 . In such cases, it is then the precise SNR distribution that determines the capacity and in a small indoor environment, this may be very similar for both clustering scenarios. Given the lower installation cost of the case with fewer clusters, this may be a preferable option in commercial indoor DAS deployments.

\section{ACKNOWLEDGEMENTS}

The authors would like to thank the U.K. Engineering and Physical Sciences Research Council (EPSRC) and the Quaternian project, funded by the EU 7th Framework Programme, for their support.

\section{REFERENCES}

[1] "Cisco Visual Networking Index : Global Mobile Data Traffic Forecast Update , 2012 2017," Cisco Systems, Tech. Rep., 2012.

[2] T. Norman, "Research forecast report - Wireless network traffic 2010-2015: forecasts and analysis," Analysys Mason, Tech. Rep., 2010.

[3] "Femtocells and Distributed Antenna Systems Complementary or Competitive?" ABI Research, Tech. Rep., 2009.

[4] M. Alatossava, A. Taparugssanagorn, V.-M. Holappa, and J. Ylitalo, "Measurement Based Capacity of Distributed MIMO Antenna System in Urban Microcellular Environment at $5.25 \mathrm{GHz}$,' in VTC Spring 2008 - IEEE Vehicular Technology Conference. IEEE, May 2008, pp. $430-434$.

[5] G. Gordon, M. Crisp, R. Penty, and I. White, "Experimental Evaluation of Layout Designs for 3x3 MIMO-Enabled Radio-overFiber Distributed Antenna Systems," IEEE Transactions on Vehicular Technology, no. c, pp. 1-1, 2013.

[6] G. S. D. Gordon, M. J. Crisp, R. V. Penty, and I. H. White, "Experimental Investigation of Antenna Selection and Transmit Beamforming for Capacity Enhancement in," in International Topical Meeting on Microwave Photonics 2013, 2013.

[7] H. Yang and T. L. Marzetta, "Performance of Conjugate and Zero-Forcing Beamforming in Large-Scale Antenna Systems," IEEE Journal on Selected Areas in Communications, vol. 31, no. 2, pp. 172-179, Feb. 2013.

[8] P. Chow, A. Karim, V. Fung, and C. Dietrich, "Performance advantages of distributed antennas in indoor wireless communication systems," Proceedings of IEEE Vehicular Technology Conference (VTC), no. 2, pp. 1522-1526, 1994.

[9] W. Feng, Y. Li, J. Gan, S. Zhou, J. Wang, and M. Xia, "On the Deployment of Antenna Elements in Generalized Multi-User Distributed Antenna Systems," Mobile Networks and Applications, vol. 16, no. 1, pp. 35-45, Oct. 2009.

[10] A. Paulraj and W. Roh, "MIMO channel capacity for the distributed antenna," in Proceedings IEEE 56th Vehicular Technology Conference, vol. 2, no. 650. IEEE, 2002, pp. 706-709.

[11] D. Tse and P. Viswanath, Fundamentals of Wireless Communication. Cambridge: Cambridge University Press, 2005.

[12] C. Oestges and B. Clerckx, MIMO wireless communications : from realworld propagation to space-time code design, 1st ed. Amsterdam: Elsevier, 2007.

[13] A. Saleh, A. Rustako, and R. Roman, "Distributed antennas for indoor radio communications," IEEE Transactions on Communications, vol. 35, no. 12, pp. 1245-1251, Dec. 1987.

[14] "IEEE Standard for Information technology Telecommunications and information exchange between systems Local and metropolitan area networks Specific requirements Part 11: Wireless LAN Medium Access Control (MAC) and Physical Layer (PHY) Specifications Am,” 2009. 